\title{
Embedded Wireless Video Grain Insect Monitoring Network
}

\author{
Peikai Yang ${ }^{1, \mathrm{a}}$, Xiong $\mathrm{Shi}^{1, \mathrm{~b},{ }^{*}}$ and $\mathrm{Lin} \mathrm{Li}^{1, \mathrm{c}}$ \\ ${ }^{1}$ School of Electric and Electronic Engineering, Wuhan Polytechnic \\ University, Wuhan, China \\ a278851511@qq.com, ${ }^{b}$ stonehero@163.net, ${ }^{c}$ lilin198509@126.com \\ *Corresponding author
}

\begin{abstract}
.
This article designed a wireless video grain insect monitoring network with wireless network sensor nodes embedded in bulk grain. This sensor node is composed of miniature camera, wireless transceiver, lighting LED, trapping device for grain insects and charging battery. The grain insects are lured into the monitoring area by the temptation of bait leading into being trapped in the help of LED light, sensor nodes with built-in camera periodically or under order takes the grain insects pictures, then the pictures are sent to the control center by wireless transmission, and finally shown in the computer screen, the real time monitoring on grain insects of quantity and species in bulk grain is achieved.
\end{abstract}

Keywords: Wireless video transmission; Grain insect monitoring; Network node.

\section{Introduction}

The grain insect problem causes severe damage to grain storage, the inspection on grain insects is to timely grasp the information about their species, intensity, distribution and damage, combining the characteristic of insects, the trend of insect damage can be predicted, a scientific proof on making decision for pest prevention and treatment is fulfilled. Nowadays the common pests inspection methods used in grain storage process include intuitive inspection method, sampling inspection method, trap detection etc, these traditional grain insect inspection technologies mainly operate or maintain complicatedly, cost large amount of workload, need improving. 
In this paper, an embedded wireless video grain insect monitoring network is designed based on video senor technology and wireless sensor technology, this network is configured with several network sensor nodes embedded in bulk grain and one or more transmitting sensor nodes (main nodes) allocated in each grain depot. Its network sensor node is composed of miniature camera, wireless transceiver, lighting LED, trapping device for grain insects and charging battery. With LED timely or randomly flashes, diffusing of bait in trapping device lures the grain insects moving into shot area of camera set in sensor node, pictures are taken at regular term or under demand, then these pictures are sent to control center in wireless transmission, which are finally shown on computer screen, the real time monitoring on grain insects of quantities and species in grain depot is achieved. This system comparing to traditional grain insects inspection methods is simpler and easier on operation, higher work efficiency. With the help of grain insect image automatic recognition software, this system can achieve automatic recognition on grain insect species and automatic statistic on grain insect distribution.

\section{System structure and Working principle}

Traditional grain insect inspection method is artificially drawing about one kilogram grain near inspection spot with drawing device, separating grain with insects by sifting device, then inspecting grain insects on species and quantity with naked eyes. With the development of electronic technology, the inspection method is improving. At present, the common inspecting method is by drawing device drawing some grain near inspection spot and transporting them on conveyer with camera above the conveyer taking pictures, the pictures are sent to computer for image recognition based on intelligent recognition technology. There is also an inspecting method in the help of built-in camera on a pole inserted in the bulk grain with wiring connection for image data collection, this wire connection is easy to be damaged by the severe corrosive fumigating agents during fumigating process of grain depot. 
In the wireless video grain insects inspecting network designed in this paper, the embedded in bulk grain of image collection sensor nodes with sealed and anti-corrosive electric circuit and in wireless transmission makes it is convenient to be put in any required position, meanwhile to transmit the pictures to computer and identified based on intelligent recognition technology, this method avoids drawing samples artificially and relieves the workload.

The wireless video grain insect monitoring network is configured with image collection nodes, relay nodes (main nodes) as well as remote controlled computer. The main nodes aggregate the data collected by video sensor nodes, and transmit these data to computer which is the destination of all video data. The system frame of wireless video grain insect monitoring network is shown in figure 1.

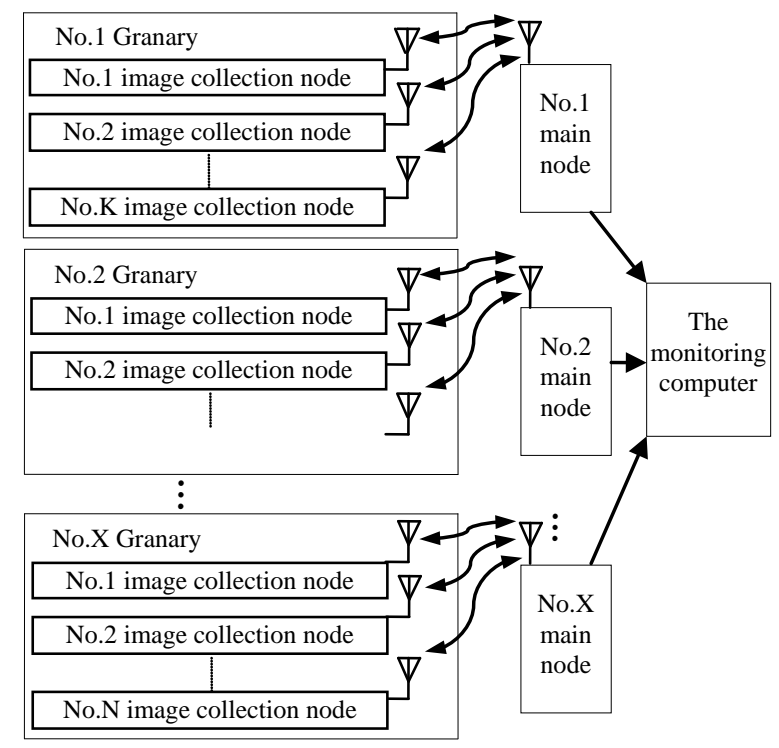

Figure 1. System Frame of Wireless Video Grain Insect Monitoring Network 


\section{Design of Image Collection Nodes (Network Nodes)}

The image collection node is composed of camera module, storage module, single-chip module integrated with radio frequency transceiving function, lithium power, light source module, sealed cover and transparent shell. The structure is shown in figure 2.

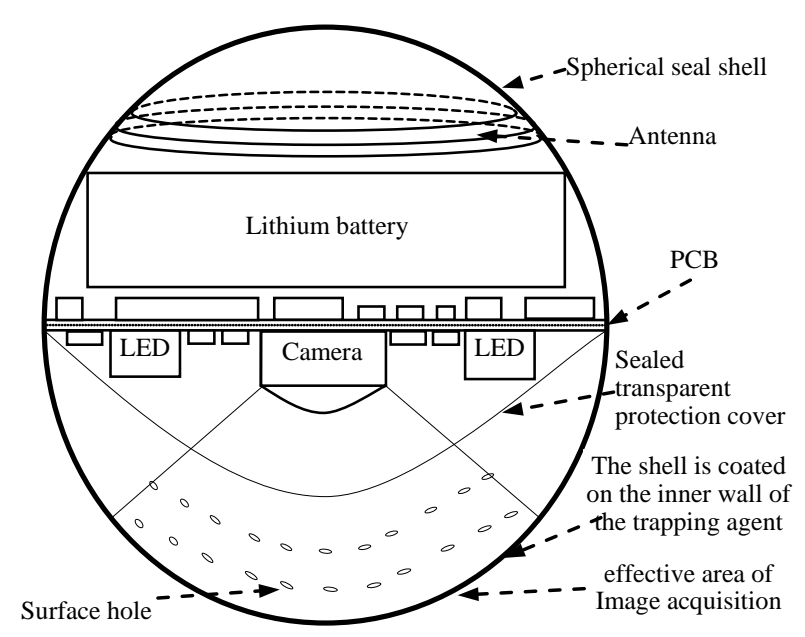

Figure 2. Composition Structure of Image Collection Sensor Node

The image collection node is designed in the ball shape with diameter about $40 \mathrm{~mm}$, its size resembles a table tennis ball, the node shell is made of anti-corrosive plastic material and sealed tightly. The hemisphere where the camera and LED located is made of transparent material, an open hole is set on the shell in the camera focusing area where the pictures of grain insects can be sharply taken, the transparent seal layer inside this hemisphere is accordingly designed to protect the camera, LED and printed circuit board from corroding by fumigating agents. 
The picture information taken by the camera is sent to single-chip through buffer storage, then through internal integrated wireless transmission module of single-chip, transmits outwards to main nodes in wireless transmission mode. The composition frame of electric circuit of image collection node is shown in figure 3.

The camera module adopts camera with OV9655 image sensor chip as the core, backing 1.3 megapixel picture taken, with highest resolution of $1280 * 1024 .{ }^{[3]}$ The size of a picture in Raw GRB output format is $1.25 \mathrm{M} \times 8 \mathrm{bit}$, if data output in speed of $20 \mathrm{k}$, several dozen seconds are needed for transmitting a picture. In the "grain and oil storage technical specification" for grain status inspection, at most one time of sampling is taken every week, that is, the real time image collection and transmission in the grain insect inspection process is not highly demanded, taking a lower transmission speed is enough.

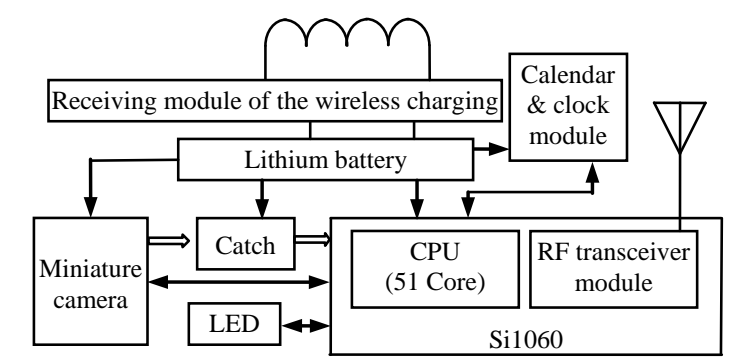

Figure 3. Diagram of Image Collection Sensor Node’s Electric Circuit The cache module adopts AL422B FIFO chip produced by Averlogic Technologies Company. The capacity of AL422B FIFO buffer chip is $384 \mathrm{~K} \times 8 \mathrm{bit}{ }^{[4]}$. The cascading of four pieces of AL422B can achieve whole data storage of $1.25 \mathrm{M} \times 8 \mathrm{bit}$. By line synchronizing signal HREF of OV9655 on piece selection of four AL422B pieces, data is read by controller from pieces of AL422B in sequence.

When a certain amount of grain insects are detected, fumigating will be taken to get rid of the insects. To avoid the corrosion to electric circuit of image 
collection sensor nodes, electric circuit must be sealed properly. The sensor node is powered by battery, with sealed electric circuit design, it is unable to change the battery, system power restraint exists. Considering the wireless sensor network in grain depot of its working characteristic and power supply, a scheme of adopting bqTESLA of TI wireless power charging is taken on the sensor nodes, after recycling the network sensor nodes, charging the batteries of sensor nodes with wireless power to solve the power problem. Meanwhile, taking strategy to lower power consumption for example in dormancy mechanism is also necessary.

As the image collection sensor node is embedded in the bulk grain, the optical source is required. Installing LED near the camera and controlling LED switch by CPU. LED as the light source also acting as luring the grain insects to be trapped. A hole is opened on the shell of image collection sensor node in the effective shot area for image collection, using the characteristic of grain insect apt to climbing the hole and aiming to light, combining the temptation of bait inside the shell to trap the grain insects.

The controller and RF module can choose wireless single-chip SI1060 from Silicon labs company, which is internal integrated high speed micro-controller and SI4432 wireless RF transceiver ${ }^{[5]}$.

\section{Design of Relay Nodes (Main Nodes)}

Main node receives the data sent by image collection node and transmits to computer, using expanded storages to achieve data access, and achieving uplink communication and downlink management. The information transmission between main nodes and computer can achieve in modes of wiring or wireless serial communication, network communication according to distribution of section areas of grain depot.

SI1060 can achieve wireless transceiving function only by setting up simple electric circuit on the surrounding. The composition frame of main node electric circuit is shown in figure 4 . 


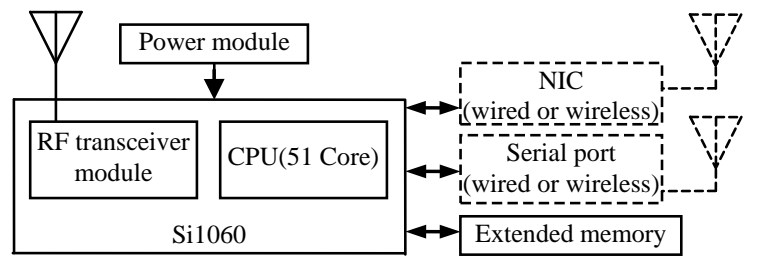

Figure 4. Composition Frame of Electric Circuit of Main Sensor Node

\section{Communication Protocol}

Monitoring computer is not only the destination of all image data, but also the control center of network. Considering normal grain depot with limited barns and no less than 10 network sensor nodes in each barn, that is, the number of network sensor nodes is not big, and taking picture sampling intervals is rather long, with enough time for image data transmission, so the work mode called roll-call polling is chosen for the network transmission process.

First numbering the network sensor nodes inside the barn in a certain sequence, monitoring computer takes turns to send query command to each network sensor node, the network sensor node replies or uploads image data through information channel after receiving the command. Being enquired node after receiving the query command either sends the image information or replies no message needs to be uploaded. The computer after receiving the reply from responsive node moves on enquiring the next node. With the clock and calendar chip set in the network sensor node, the system can set picture shot time for each network sensor node to automatic shot and upload picture, proper time set required in need of avoiding two or more nodes sending pictures at the same time causing the transmission channel conflict.

\section{Conclusion}


By embedded wireless video grain insect monitoring network can grasp real time grain insect information in grain depot, providing scientific proof with accurate data for grain pest prevention and treatment, reducing the loss from grain pest in grain storage. China is grain industry giant, there are huge application market and broad developing prospect in using the wireless video sensor network technology.

\section{Acknowledgement}

This paper is funded by scientific research project of Wuhan Polytechnic University (Project number: 2013d19).

\section{References}

[1] Xuguang BAI, Storage Pest Prevention and Treatment, Science Press, Beijing, 2008(In Chinese)

[2] Li Tan, Wireless Sensor Network Theory and Technology Implement. China Machine Press, Beijing, 2011(In Chinese)

[3] OmniVision Technologies.OV9655 Datasheet [M]. USA: Omni Vision Technologies, 2006.

[4] Averlogic Technologies.AL422B Datasheet [M]. USA: Averlogic Technologic, 2001.

[5] Silicon Labs, Si106x/108x Datasheet[M].USA: Silicon Laboratories,2014. 